\title{
The value of trichoscopy in the differential diagnosis of scalp lesions in pemphigus vulgaris and pemphigus foliaceus*
}

\author{
Marta Sar-Pomian ${ }^{1}$ \\ Lidia Rudnicka ${ }^{1,2}$
}

\author{
Marta Kurzeja ${ }^{1}$ \\ Malgorzata Olszewska ${ }^{1}$
}

DOI: $h$ ttp://dx.doi.org/10.1590/abd1806-4841.20143830

\begin{abstract}
BACKGROUND: Trichoscopy is becoming increasingly popular in diagnosing hair and scalp diseases. Scalp involvement in pemphigus is common. The scalp may be the first or only site of clinical manifestation of the disease. ОвјестіVE: The aim of this study was to analyze whether trichoscopy may be useful in aiding differential diagnosis of scalp lesions in patients with pemphigus vulgaris and pemphigus foliaceus.

METHODS: Trichoscopy was performed in 19 patients with scalp lesions in the course of pemphigus ( 9 patients with pemphigus vulgaris and 10 with pemphigus foliaceus). In all patients, the diagnosis of scalp pemphigus was confirmed by histopathology. The working magnification was 20 -fold and 70 -fold.

RESULTS: The most frequently observed trichoscopy features of pemphigus lesions were: extravasations (18/19; $94.7 \%)$ and yellow hemorrhagic crusts (11/19; 57.9\%). Yellow dots with whitish halo were observed in 6/19 $(31.6 \%)$ patients with pemphigus. White polygonal structures were observed in pemphigus foliaceus $(6 / 10 ; 60 \%)$, but not in pemphigus vulgaris. Vascular abnormalities were more frequent in pemphigus vulgaris, when compared to pemphigus foliaceus, and were associated with a severe course of disease. Linear serpentine vessels were the most frequent vascular abnormality in patients with pemphigus vulgaris and pemphigus foliaceus $(77.8 \%$ and $30 \%$, respectively).

CONCLUSION: Trichoscopy may serve as a useful supplementary method in the differential diagnosis of pemphigus, especially in cases of desquamative or exudative lesions limited to the scalp. Extravasations, yellow hemorrhagic crusts, yellow dots with whitish halo, white polygonal structures and linear serpentine vessels are trichoscopy features which may suggest the diagnosis of pemphigus.
\end{abstract}

Keywords: Dermoscopy; Desmoglein 1; Desmoglein 3; Hair; Hair follicle; Pemphigus

\section{INTRODUCTION}

Pemphigus is a rare, potentially life-threatening autoimmune vesiculobullous disease affecting the skin and mucosa. It is characterized by the presence of circulating and in vivo-bound autoantibodies directed against desmogleins. ${ }^{1,2}$ Pemphigus vulgaris (PV) is characterized by blisters and erosions of the skin and/or mucous membranes, and circulating antibodies directed against desmoglein 3 and $1{ }^{1,2}$ In pemphigus foliaceus (PF), there is only skin involvement, without mucosal lesions, and antibodies are exclusively directed against desmoglein $1 .^{3}$

Scalp involvement is often observed in patients with pemphigus vulgaris and pemphigus foliaceus. ${ }^{4,5}$ Frequently, the scalp is the first site of clinical manifestation of the disease., ${ }^{4,6}$ Isolated pemphigus lesions of the scalp have been reported in the literature. ${ }^{7-9}$ Occasionally, scalp lesions may be extremely resistant to treatment. ${ }^{10,11}$ Scalp lesions in the course of pemphigus can lead to several types of non-cicatricial and cicatricial alopecia. ${ }^{6,12-14}$ Thus, differential diagnosis of isolated scalp lesions and hair loss in the course of pemphigus

Trichoscopy (hair and scalp dermoscopy) is a useful tool in the differential diagnosis of hair loss and inflammatory scalp diseases. ${ }^{15}$ It is a non-invasive technique in which either a handheld dermoscope or a digital videodermoscope can be used to visualize hair and scalp structures. ${ }^{16}$ The method has well-established position as an ancillary tool in the diagnosis of

Received on 10.07.2014.

Approved by the Advisory Board and accepted for publication on 06.09.2014.

* Study conducted at the Department of Dermatology, Medical University of Warsaw - Warszawa, Poland.

Conflict of interest: None

Financial funding: None

Medical University of Warsaw -Department of Dermatology - Warsaw, Poland.

Mossakowski Medical Research Center, Polish Academy of Sciences - Department of Neuropeptides - Warsaw, Poland.

C 2014 by Anais Brasileiros de Dermatologia

An Bras Dermatol. 2014;89(6):1007-12. 
such disorders as tinea capitis, alopecia areata, androgenetic alopecia, discoid lupus erythematosus, lichen planopilaris, folliculitis decalvans and other hair and scalp diseases. ${ }^{17-27}$ However, according to our literature search, there is only one case report on dermoscopy of pemphigus. ${ }^{28}$ The aim of our study was to evaluate trichoscopy features of scalp lesions in patients with pemphigus vulgaris and pemphigus foliaceus, and to establish the potential value of this method in the differential diagnosis of pemphigus.

\section{MATERIAL AND METHODS}

A total of 19 patients with scalp involvement in the course of pemphigus were included into the study (9 patients with pemphigus vulgaris and 10 with pemphigus foliaceus). The diagnosis of pemphigus was based on physical examination, histopathology of skin lesions, direct and indirect immunofluorescence results, and presence of anti-desmoglein 1 or 3 antibodies in enzyme-linked immunosorbent test (ELISA). In all patients the diagnosis of scalp pemphigus was confirmed by histopathology and direct immunofluorescence.

Patients with paraneoplastic pemphigus were not included into the study. ${ }^{29}$ The group of patients with PV consisted of 4 women and 5 men, aged 18-89, with pemphigus lasting 4 months to 19 years, and scalp involvement lasting from 4 months to 6 years. The group of patients with PF consisted of 3 women and 7 men, aged $44-88$ years, with pemphigus lasting from 4 months to 16 years, and scalp involvement lasting from 2 months to 11 years. The extent of scalp lesions was determined according to the Pemphigus Disease Area Index for the scalp (Table 1). ${ }^{30}$

At the time of the scalp examination, skin lesions were observed in $4 / 9(44,4 \%)$ patients with pemphigus vulgaris, whereas mucosal lesions were present in $6 / 9(66,7 \%)$ patients. Skin lesions were present in 8/10 (80\%) patients with pemphigus foliaceus. Mucosal lesions were not present in these patients.

A total of $5 / 9(55,6 \%)$ patients with PV and $5 / 10(50 \%)$ patients with PF were newly diagnosed

TABle 1: Pemphigus Disease Area Index for the scalp

\begin{tabular}{ll}
\hline Score & Erosion/Blisters or new erythema \\
\hline 0 & Absent \\
1 & In one quadrant \\
2 & In two quadrants \\
3 & In three quadrants \\
4 & Affects whole skull \\
10 & At least one lesion $>6 \mathrm{~cm}$ \\
\hline
\end{tabular}

Source: Rahbar Z, et al ${ }^{30}$ and Murrell DF, et al 45 with no previous therapy. In other cases trichoscopy was performed during a clinical and immunological recurrence in the course of immunosuppressive therapy that consisted of prednisone and azathioprine in $\mathrm{PV}$ and of prednisone in monotherapy in PF.

Trichoscopy was performed with the use of the Fotofinder 2 Videodermoscopy System, with a 20-fold and 70-fold working magnification. Trichoscopy abnormalities in patients with pemphigus were examined according to the standard protocol and definitions. ${ }^{20}$

\section{RESULTS}

In all patients with pemphigus vulgaris trichoscopy showed extravasations within the scalp lesions $(9 / 9 ; 100 \%)$ (Figure 1). Yellow hemorrhagic crusts were observed in 5/9 (55.6\%) patients. Linear serpentine vessels were observed in $7 / 9(77.8 \%)$ patients. Lace-like vessels were observed in 5/9 (55.6\%) patients. Linear helical vessels, as well as glomerular vessels and dotted vessels with whitish halo were observed less frequently, but corresponded to the highest PDAI for the scalp. Additionally, in one patient we observed an area of glomerular vessels aligned in rings, forming "a string of pearls"-like arrangement. Detailed data are presented in Table 2.

Extravasations were observed in 9/10 (90\%) patients with pemphigus foliaceus (Figure 2). Yellow hemorrhagic crusts were observed in 6/10 (60\%) patients. The most common type of vessels were linear serpentine vessels, observed in 3/10 (30\%) patients. Linear helical vessels and glomerular vessels were present in 2 patients with a PDAI score for the scalp equal to 10 .

Various types of scaling were observed in patients with pemphigus foliaceus, namely: white diffuse scaling (in all patients), white polygonal structures $(60 \%$ of patients), yellow diffuse scaling (30\%), tubular perifollicular scaling and hair casts $(20 \%)$. Yellow dots with whitish halo were observed in 2/10 $(20 \%)$ patients. The most characteristic trichoscopy findings are illustrated in Figure 3. Detailed data are presented in table 2 .

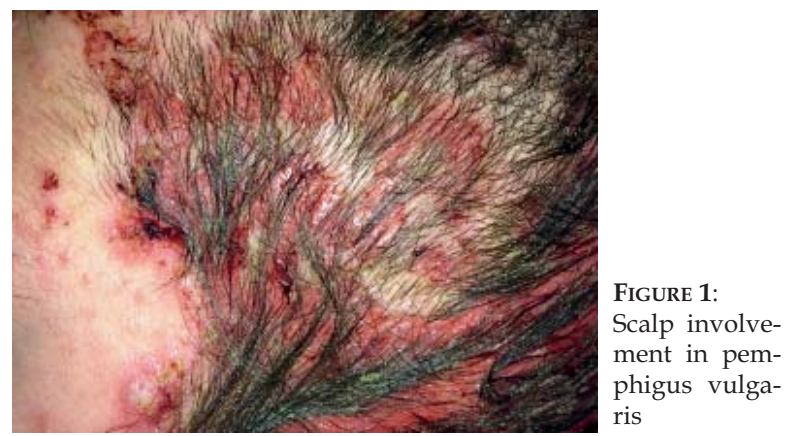


TABLE 2: Trichoscopy features of scalp lesions in patients with pemphigus vulgaris (PV) and pemphigus foliaceus (PF)

\begin{tabular}{|c|c|c|}
\hline & PV & PF \\
\hline $\operatorname{Sex}(F / M)$ & $4 / 5$ & $3 / 7$ \\
\hline Age & $58.8+/-19.7$ & $64.2+/-16.4$ \\
\hline Duration of the disease [years] & $7.3+/-10.6$ & $3.8+/-5.3$ \\
\hline Duration of the scalp involvement [years] & $2.1+/-2.4$ & $2.9+/-4.0$ \\
\hline PDAI - scalp & $4.2+/-4.4$ & $3.4+/-3.6$ \\
\hline PDAI - skin & $5.6+/-14.1$ & $15.3+/-24.9$ \\
\hline PDAI - mucous membranes & $6.9+/-14.6$ & $0 / 0$ \\
\hline Dotted vessels & $1 / 9(11.1 \%)$ & 0 \\
\hline Dotted vessels with whitish halo & $2 / 9(22.2 \%)$ & 0 \\
\hline Thin arborizing vessels & $2 / 9(22.2 \%)$ & $2 / 10(20 \%)$ \\
\hline Thick arborizing vessels & $1 / 9(11.1 \%)$ & 0 \\
\hline Yellow hemorrhagic crusts & $5 / 9(55.6 \%)$ & $6 / 10(60 \%)$ \\
\hline Extravasations & $9 / 9(100 \%)$ & $9 / 10(90 \%)$ \\
\hline Linear helical vessels & $2 / 9(22.2 \%)$ & $1 / 10(10 \%)$ \\
\hline Lace-like vessels & $5 / 9(55.6 \%)$ & $2 / 10(20 \%)$ \\
\hline Glomerular vessels & $2 / 9(22.2 \%)$ & $1 / 10(10 \%)$ \\
\hline Linear serpentine vessels & $7 / 9(77.8 \%)$ & $3 / 10(30 \%)$ \\
\hline White diffuse scaling & $4 / 9(44.4 \%)$ & $10 / 10(100 \%)$ \\
\hline Yellow diffuse scaling & $2 / 9(22.2 \%)$ & $3 / 10(30 \%)$ \\
\hline Tubular scaling & 0 & $2 / 10(20 \%)$ \\
\hline White perifollicular areas "white veil" & $3 / 9(33.3 \%)$ & 0 \\
\hline Pink areas & $2 / 9(22.2 \%)$ & 0 \\
\hline Perifollicular yellow areas & $3 / 9(33.3 \%)$ & $2 / 10(20 \%)$ \\
\hline Black dots & $1 / 9(11.1 \%)$ & 0 \\
\hline Yellow dots & $7 / 8(77.8 \%)$ & $3 / 30(30 \%)$ \\
\hline White dots & $2 / 9(22.2 \%)$ & 0 \\
\hline Yellow dots with whitish halo, 'fried egg sign' & $4 / 9(44.4 \%)$ & $2 / 10(20 \%)$ \\
\hline White dots with yellow halo 'reversed fried egg' & 0 & $2 / 20(20 \%)$ \\
\hline Hair casts & $1 / 9(11.1 \%)$ & $2 / 10(20 \%)$ \\
\hline White polygonal structures & 0 & $6 / 10(60 \%)$ \\
\hline
\end{tabular}

PDAI $=$ Pemphigus Disease Area Index

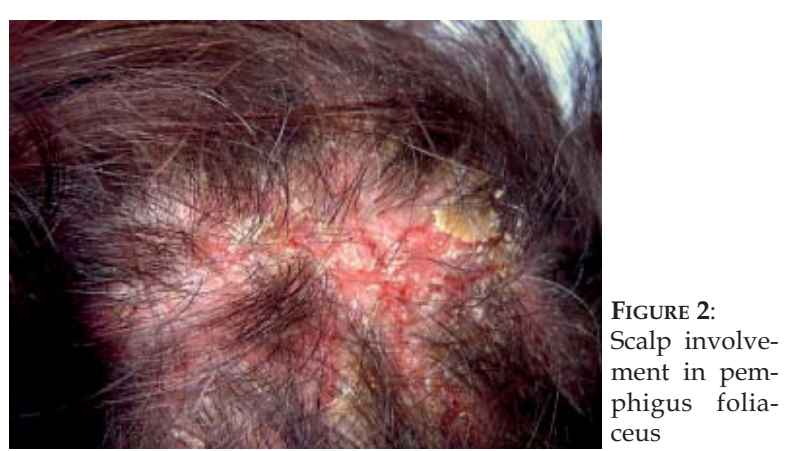

\section{DISCUSSION}

Differential diagnosis of scalp lesions in the course of pemphigus vulgaris and pemphigus foliaceus may be a diagnostic challenge, especially when they are the first or sole cutaneous manifestation of disease. Pemphigus of the scalp is clinically manifested by cutaneous inflammation with crusts and intense scaling. ${ }^{4}$ These changes may be accompanied by erosions, but blisters are usually not present. ${ }^{4}$ Less common manifestations of pemphigus of the scalp are verrucous, vegetating plaques and tufted folliculitis. ${ }^{10,31}$ These inflammatory changes are commonly associated with cicatrical or non-cicatricial alopecia. ${ }^{32,33}$ Thus, it seems apparent that trichoscopy may potentially serve as a rapid and easy method for the initial differential diagnosis of scalp lesions in such cases.

There are currently no literature data addressing the usefulness of trichoscopy (or dermoscopy in general) in the differential diagnosis of pemphigus, except a case report of hair casts in a 57-year-old patient with pemphigus vulgaris. ${ }^{28}$ Hair casts are 3-7mm-long, whitish or yellowish tubular structures which envelop the hair shafts. ${ }^{16,34}$ In this first description, Pirmez suggested that hair casts develop in pemphigus vulgaris in a mechanism associated with acantholysis within the outer root sheet. ${ }^{28}$ As the hair grows, the outer root sheath keratinocytes move up through the follicular openings to form the hair cast. This phenomenon could be considered a Nikolsky's sign in the scalp hair follicles ${ }^{12}$ and may be explained by the distribution of desmoglein 3 within outer root sheath. Desmoglein 3 is expressed throughout all 

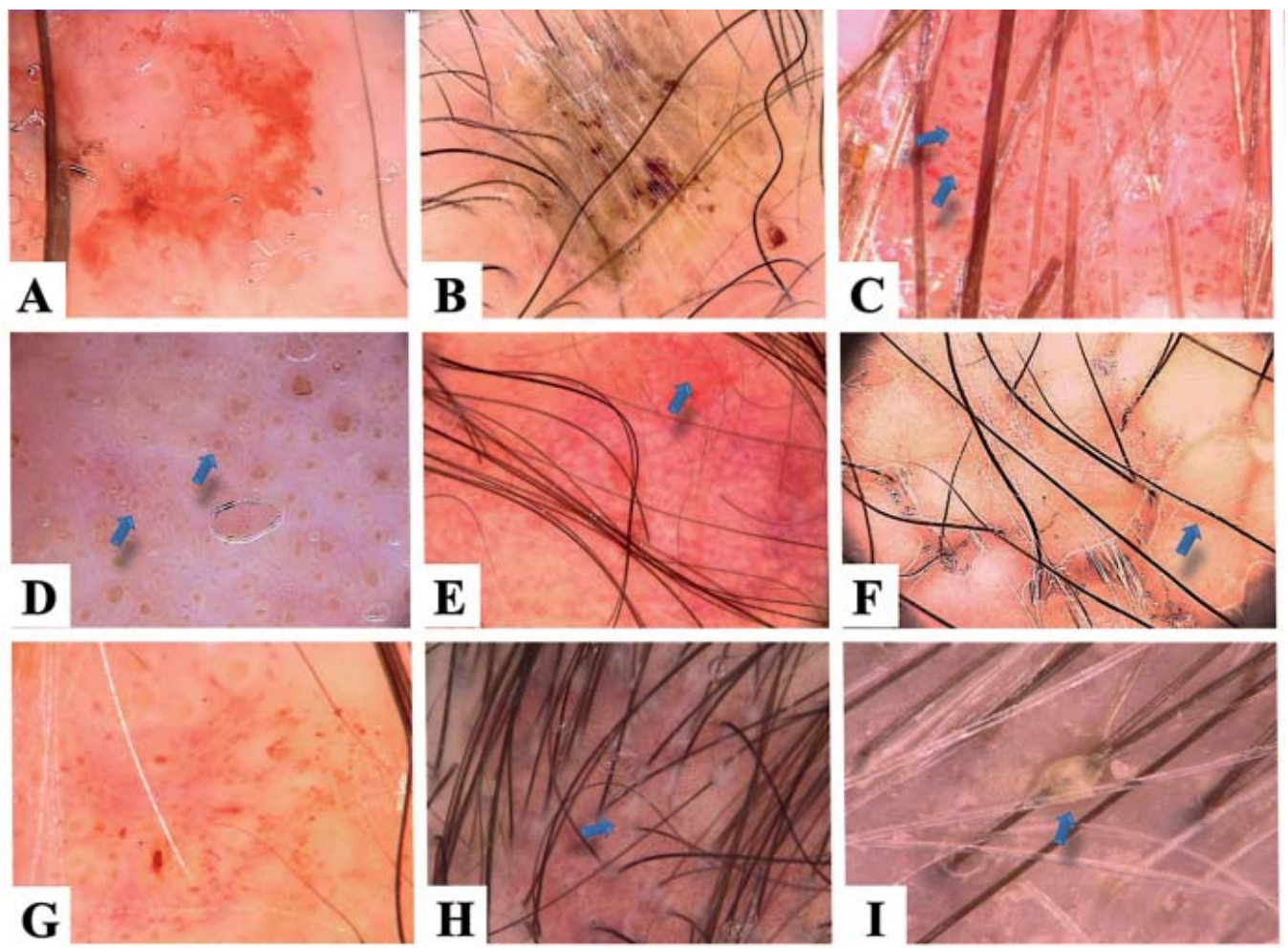

FIGURE 3:

Trichoscopy features of pemphigus: extravasations (A), yellow hemorrhagic crusts (B), serpentine vessels (C), yellow dots with whitish halo - the 'fried egg sign' (D), circular alignment of vessels (E), white polygonal structures (F), irregularly distributed blood vessels (G), tubular scaling $(\mathrm{H})$ and hair casts (I)

layers of outer root sheath, in areas of tricholemmal keratinization, and in the basal layer of the outer root sheath in areas of epidermal keratinization. ${ }^{35}$ The hypothesis of Pirmez may be confirmed by the findings of Delmonte et al ${ }^{12}$, who described three cases of pemphigus vulgaris in whom the hairs plucked from both lesional and perilesional skin were anagen hairs with intact root sheaths. In our study hair casts were observed in only $11.1 \%$ of patients with pemphigus vulgaris and in $20 \%$ of patients with pemphigus foliaceus. In all cases the presence of hair casts was associated with long-lasting diffuse scaling, but average PDAI for scalp was 4.3 , what reflects intermediate activity of scalp involvement in the course pemphigus. Our data may indicate that hair casts in pemphigus are rather a sign of severe diffuse scaling (similar to Pityriasis amiantacea) than a direct reflection of acantholysis. $^{36}$

In our study two types of hair casts could be distinguished: 1) poorly-defined, whitish, cottonwool-like hair casts in pemphigus vulgaris and 2) well-defined, yellowish or whitish hair casts in pemphigus foliaceus.

The most consistent trichoscopy findings in patients with pemphigus were extravasations, appearing as red hemorrhagic polygonal structures. This feature was observed in all patients with pemphigus vulgaris and pemphigus foliaceus. This finding is not specific for pemphigus. Extravasations may be occasionally observed in other inflammatory scalp diseases, such as psoriasis, seborrheic dermatitis, pemphigoid, pediculosis and skin cancers..$^{16,37,38}$ However, the results of this study and our experience show that an abundance of extravasations in all fields of view of a dermoscope may be indicative for an autoimmune bullous disease.

Yellow hemorrhagic crusts were observed frequently in the examined scalp lesions. The presence of yellow hemorrhagic crusts is not specific for pemphigus. It is a common trichoscopy finding in diseases associated with pus secretion, such as folliculitis decalvans or dissecting cellulitis. ${ }^{21,23}$ It may be considered whether such a trichoscopy finding in patients with pemphigus should be an indication for antibacterial treatment.

A common feature of scalp lesions in pemphigus was the presence of white diffuse scaling, occurring in $74 \%$ of patients $(44 \%$ in pemphigus vulgaris and $100 \%$ in pemphigus foliaceus). Diffuse white scaling is a non-specific finding. It may occur in psoriasis, discoid lupus erythematosus, allergic dermatitis and dry skin. ${ }^{39,40}$ Yellow diffuse scaling was slightly more common in patients with pemphigus foliaceus, when compared to pemphigus vulgaris $(40 \%$ and $22.2 \%$, respectively). Yellow diffuse scaling also occurs in seborrheic dermatitis, discoid lupus erythematosus 
and ichthyosis. ${ }^{22,41,42}$ In $60 \%$ of patients with pemphigus foliaceus, the scaling had an appearance of white polygonal structures. Polygonal white structures were not observed in pemphigus vulgaris and were not previously described in other scalp disorders in the literature. This is a novel finding in our study.

In patients with pemphigus vulgaris, trichoscopy with immersion fluid revealed white cotton- woollike areas, which correlated positively with the extension of the scalp involvement (PDAI 10). These type of structures have been shown to be a trichoscopy manifestation of scales observed with immersion fluid. ${ }^{16}$

Another common trichoscopy feature of scalp pemphigus was the presence of yellow dots. Yellow dots are a trichoscopy manifestation of empty hair follicles filled with keratinous material and sebum..$^{27,43}$ In our study yellow dots were observed more frequently in pemphigus vulgaris $(77.8 \%)$, when compared to pemphigus foliaceus (40\%).

A novel trichoscopy finding in this study was the detection of yellow dots with a whitish halo. It may be hypothesized that these are empty hair follicles surrounded by an acanthotic outer root sheath. This trichoscopy finding has not been previouly described in the literature. We suggest that this finding is referred to as "fried egg sign". It is specific for pemphigus. A reversed version of this sign (white dots with yellow halo) were observed only in $20 \%$ of patients and only in pemphigus foliaceus.

Vascular abnormalities were more frequently observed in patients with pemphigus vulgaris, when compared to pemphigus foliaceus. Linear serpentine vessels occurred in pemphigus vulgaris and pemphi- gus foliaceus in $77.8 \%$ and $40 \%$ of patients, respectively. Moreover, the presence of vascular abnormalities (especially linear helical vessels and glomerular vessels) correlated with high Pemphigus Disease Area Index for the scalp and severe course of the disease. There are no literature data on dermoscopic or trichoscopic features of blood vessels in pemphigus. However, Kurzeja et al described vasodilatation in reflectance confocal microscopy of the upper dermis in $61 \%-86 \%$ of cutaneous lesions in patients with pemphigus. ${ }^{44}$

\section{CONCLUSION}

In conclusion, trichoscopy is a useful tool in the differential diagnosis of pemphigus, especially in cases of desquamative and exudative lesions limited to the scalp. Immunological examinations are the gold standard for diagnosing pemphigus, but trichoscopy may serve as a non-time consuming technique that may facilitate the initial diagnosis and help choosing an optimal site to obtain a biopsy specimen. ${ }^{45}$ Extravasations and yellow hemorrhagic crusts were the most frequent findings in scalp lesions of patients with pemphigus. However, the "fried egg sign" was identified as a new, trichoscopy- specific feature of pemphigus.]

\section{ACKNOWLEDGEMENTS}

The study has been supported by the research grant No NN402371538 from the National Science Centre in Poland.

The authors thank Dr. Maria Victória Quaresma for her help in preparing the abstract in Portuguese. 


\section{REFERENCES}

1. Cunha PR, Barraviera SR. Autoimmune bullous dermatoses. An Bras Dermatol. 2009;84:111-24.

2. Porro AM, Caetano Lde V, Maehara Lde S, Enokihara MM. Non-classical forms of pemphigus: pemphigus herpetiformis, IgA pemphigus, paraneoplastic pemphigus and $\lg G / \lg A$ pemphigus. An Bras Dermatol. 2014;89:96-106.

3. Ruocco V, Ruocco E, Lo Schiavo A, Brunetti G, Guerrera LP, Wolf R. Pemphigus: etiology, pathogenesis, and inducing or triggering factors: facts and controversies. Clin Dermatol. 2013;31:374-81.

4. Sar-Pomian M, Kolacinska-Strasz Z, Labecka H, Krainska-Wojcik T, Olszewska M. Scalp lesions in pemphigus. Przegl Dermatol. 2010;97:14-20.

5. Oretti G, Giordano D, Di Lella F, Gradoni P, Zendri E, Ferri T. Unilesional pemphigus vulgaris of the scalp after cochlear implantation. Am J Otolaryngol. 2011;32:80-1.

6. Hadayer N, Ramot Y, Maly A, Zlotogorski A. Pemphigus vulgaris with loss of hair on the scalp. Int J Trichology. 2013;5:157-8.

7. Lehrhoff S, Miller K, Fischer M, Kamino H, Meehan S. Localized pemphigus with vegetative features. Dermatol Online J. 2012;18:11.

8. Yamamoto S, Kanekura T, Gushi A, Sekiyama M, Shimada T, Shimada K, et al. A case of localized pemphigus foliaceus. J Dermatol. 1996;23:893-5.

9. Lapière K, Caers S, Lambert J. A case of long-lasting localized pemphigus vulgaris of the scalp. Dermatology. 2004;209:162-3.

10. Ko DK, Chae IS, Chung KH, Park JS, Chung H. Persistent pemphigus vulgaris showing features of tufted hair folliculitis. Ann Dermatol. 2011;23:523-5.

11. Olszewska M, Kolacinska-Strasz Z, Sulej J, Labecka H, Cwikla J, Natorska U, et al. Efficacy and safety of cyclophosphamide, azathioprine, and cyclosporine (ciclosporin) as adjuvant drugs in pemphigus vulgaris. Am J Clin Dermatol. 2007;8:85-92.

12. Delmonte S, Semino MT, Parodi A, Rebora A. Normal anagen effluvium: a sign of pemphigus vulgaris. Br J Dermatol. 2000;142:1244-5

13. Petronić-Rosić V, Krunić A, Mijusković M, Vesić S. Tufted hair folliculitis: a pattern of scarring alopecia? J Am Acad Dermatol. 1999 Jul;41:112-4.

14. Saijyo S, Tagami H. Tufted hair folliculitis developing in a recalcitrant lesion of pemphigus vulgaris. J Am Acad Dermatol. 1998;38:857-9.

15. Rudnicka L, Olszewska M, Rakowska A, Kowalska-Oledzka E, Slowinska M. Trichoscopy: a new method for diagnosing hair loss. J Drugs Dermatol. 2008;7:651-4.

16. Rudnicka L, Olszewska M, Rakowska A. Atlas of trichoscopy: dermoscopy in hair and scalp disease. London: Springer; 2012.

17. Slowinska M, Rudnicka L, Schwartz RA, Kowalska-Oledzka E, Rakowska A, Sicinska J, et al. Comma hairs: a dermatoscopic marker for tinea capitis: a rapid diagnostic method. J Am Acad Dermatol. 2008:59:S77-9

18. Pinheiro AM, Lobato LA, Varella TC. Dermoscopy findings in tinea capitis: case report and literature review. An Bras Dermatol. 2012;87:313-4.

19. Abraham LS, Torres FN, Azulay-Abulafia L. Dermoscopic clues to distinguish trichotillomania from patchy alopecia areata. An Bras Dermatol. 2010;85:723-6.

20. Rakowska A, Slowinska M, Kowalska-Oledzka E, Olszewska M, Rudnicka L. Dermoscopy in female androgenic alopecia: method standardization and diagnostic criteria. Int J Trichology. 2009;1:123-30.

21. Rakowska A, Slowinska M, Kowalska-Oledzka E, Warszawik 0, Czuwara J, Olszewska $\mathrm{M}$, et al. Trichoscopy of cicatricial alopecia. J Drugs Dermatol. 2012:11:753-8.

22. Duque-Estrada B, Tamler C, Sodré CT, Barcaui CB, Pereira FB. Dermoscopy patterns of cicatricial alopecia resulting from discoid lupus erythematosus and lichen planopilaris. An Bras Dermatol. 2010;85:179-83.

23. Fabris MR, Melo CP, Melo DF. Folliculitis decalvans: the use of dermatoscopy as an auxiliary tool in clinical diagnosis. An Bras Dermatol. 2013;88:814-6.

24. Rudnicka L, Olszewska M, Rakowska A, Slowinska M. Trichoscopy update 2011. J Dermatol Case Rep. 2011;5:82-8.

25. Oliveira LC, Miranda AR, Pinto SA, lanhez M. Case for diagnosis. An Bras Dermatol. 2014;89:353-5.

26. Pirmez R, Piñeiro-Maceira J, Almeida BC, Sodré CT. Follicular red dots: a normal trichoscopy feature in patients with pigmentary disorders? An Bras Dermatol. 2013:88:459-61.

27. Torres F, Tosti A. Trichoscopy: an update. G Ital Dermatol Venereol. 2014;149:83-91.

28. Pirmez R. Acantholytic hair casts: a dermoscopic sign of pemphigus vulgaris of the scalp. Int J Trichology. 2012;4:172-3.
29. Silva JA, Mesquita Kde C, Igreja AC, Lucas IC, Freitas AF, Oliveira SM, et al Paraneoplastic cutaneous manifestations: concepts and updates. An Bras Dermatol. 2013:88:9-22.

30. Rahbar Z, Daneshpazhooh M, Mirshams-Shahshahani M, Esmaili N, Heidari K, Aghazadeh N, et al. Pemphigus disease activity measurements: pemphigus disease area index, autoimmune bullous skin disorder intensity score, and pemphigus vulgaris activity score. JAMA Dermatol. 2014;150:266-72.

31. Danopoulou I, Stavropoulos P, Stratigos A, Chatziolou E, Chiou A, Georgala S, et al. Pemphigus vegetans confined to the scalp. Int J Dermatol. 2006;45:1008-9.

32. Veraitch 0 , Ohyama M, Yamagami J, Amagai M. Alopecia as a rare but distinct manifestation of pemphigus vulgaris. J Eur Acad Dermatol Venereol. 2013;27:86-91.

33. Gaitanis G, Patmanidis K, Skandalis K, Alexis I, Zioga A, Bassukas ID. Scaring alopecia in pemphigus vulgaris: a rare or underdiagnosed presentation? Eur J Dermatol. 2013;23:253-5.

34. França K, Villa RT, Silva IR, de Carvalho CA, Bedin V. Hair casts or pseudonits. Int J Trichology. 2011;3:121-2

35. Wu H, Stanley JR, Cotsarelis G. Desmoglein isotype expression in the hair follicle and its cysts correlates with type of keratinization and degree of differentiation. $J$ Invest Dermatol. 2003;120:1052-7.

36. Verardino GC, Azulay-Abulafia L, Macedo PM, Jeunon T. Pityriasis amiantacea: clinical-dermatoscopic features and microscopy of hair tufts. An Bras Dermatol. 2012;87:142-5.

37. Martins LG, Bernardes Filho F, Quaresma MV, Bellott TR, Botelho LN, Prata AC Dermoscopy applied to pediculosis corporis diagnosis. An Bras Dermatol. 2014;89:513-4.

38. Kardynal A, Olszewska M. Modern non-invasive diagnostic techniques in the detection of early cutaneous melanoma. J Dermatol Case Rep. 2014:8:1-8.

39. Kim KS, Shin MK, Ahn JJ, Haw CR, Park HK. Investigation of hair shaft in seborrheic dermatitis using atomic force microscopy. Skin Res Technol. 2011;17:288-94.

40. Miteva M, Tosti A. Dermatoscopy of hair shaft disorders. J Am Acad Dermatol. 2013;68:473-81.

41. Haliasos EC, Kerner M, Jaimes-Lopez N, Rudnicka L, Zalaudek I, Malvehy J, et al Dermoscopy for the pediatric dermatologist part I: dermoscopy of pediatric infectious and inflammatory skin lesions and hair disorders. Pediatr Dermatol. 2013;30:163-71

42. Haliasos EC, Kerner M, Jaimes N, Zalaudek I, Malvehy J, Lanschuetzer CM, et al. Dermoscopy for the pediatric dermatologist, part ii: dermoscopy of genetic syndromes with cutaneous manifestations and pediatric vascular lesions. Pediatr Dermatol. 2013;30:172-81.

43. Ross EK, Vincenzi C, Tosti A. Videodermoscopy in the evaluation of hair and scalp disorders. J Am Acad Dermatol. 2006;55:799-806.

44. Kurzeja M, Rakowska A, Rudnicka L, Olszewska M. Criteria for diagnosing pemphigus vulgaris and pemphigus foliaceus by reflectance confocal microscopy. Skin Res Technol. 2012;18:339-46.

45. Murrell DF, Dick S, Ahmed AR, Amagai M, Barnadas MA, Borradori L, et al. Consensus statement on definitions of disease, end points, and therapeutic response for pemphigus. J Am Acad Dermatol. 2008;58:1043-6.

MAILING ADDRESS:
Malgorzata Olszewska
Department of Dermatology
Medical University of Warsaw
Koszykowa 82A
02-008 Warsaw, Poland
E-mail: malgorzata.olszewska@wum.edu.pl

How to cite this article: Sar-Pomian M, Kurzeja M, Rudnicka L, Olszewska M. The value of trichoscopy in the differential diagnosis of scalp lesions in pemphigus vulgaris and pemphigus foliaceus. An Bras Dermatol. 2014;89(6):1007-12. 